\title{
How Critical is the Duration of the Sampling Scheme for the Determination of Half-Life, Characterization of Exposure and Assessment of Bioequivalence?
}

\author{
Philippe Colucci ${ }^{1,2} ;$ Jacques Turgeon $^{1,3}$; and Murray P. Ducharme ${ }^{1,2}$ \\ 1. Faculté de Pharmacie, University of Montreal, Montreal, Canada. \\ 2. Cetero Research, Cary, North Carolina, USA. \\ 3. CRCHUM, Centre Hospitalier de l'Université de Montréal, Montreal, Canada.
}

Received, December 22, 2010; Revised, March 28, 2011; Accepted, May 27, 2011; Published, May 28, 2011

\begin{abstract}
In noncompartmental analysis, poor characterization of the terminal elimination rate constant $(\mathrm{Kel})$ will lead to biased results for half-life and total exposure (AUCinf), providing incorrect relative bioavailability and bioequivalence conclusions. We set out to determine if the sampling scheme duration was crucial for proper half-life and AUCinf determination. Profiles for 1000 subjects were simulated with a sampling scheme covering five half-lives. Concentrations were gradually removed from the end of the profile to determine if precision and bias in the half-life and AUCinf values were affected. Additionally, 30 bioequivalence studies were simulated to determine the influence of unreliable AUCinf PK parameter on BE conclusions. Precision and bias became unacceptable for AUCinf and half-life if Kel was not determined with a sampling scheme covering at least 2 and 4 half-lives, respectively. Bioequivalence conclusions also deteriorated if unreliable PK parameters were maintained. Sampling scheme duration is important when calculating noncompartmental parameters. In conclusion, sampling scheme duration should be at least 4 times the average measured half-life in order to have confidence in the reported half-life values. Additionally, individual subject's pharmacokinetic parameters should be removed from the pivotal statistical analysis when their associated calculated half-life is longer than half of the total sampling interval.
\end{abstract}

This article is open to POST-PUBLICATION REVIEW. Registered readers (see "For Readers") may comment by clicking on ABSTRACT on the issue's contents page.

\section{INTRODUCTION}

Bioequivalence (BE) studies usually assess and compare the rate and extent to which the active ingredient or active moiety becomes available into the systemic circulation.[1] Theoretically, if two formulations of the same active product produce similar systemic concentration profiles, they will also produce similar concentration profiles at the site of action and therefore produce comparable pharmacological effects.[1,2]

To properly characterize relative bioavailability (BA) or establish BE in terms of extent of exposure, total exposure is measured by the PK parameter area under the curve from time zero to infinity (AUCinf).[3] This parameter is calculated as the area under the curve from time zero to the last measurable concentration (AUC0-t) plus the last measurable drug concentration divided by the terminal elimination rate constant $\left(\lambda_{z}\right.$ or Kel). $[4,5]$
Therefore, in order to properly characterize AUCinf, the terminal rate constant and terminal elimination half-life $\left(\mathrm{T}^{1} / 2\right)$ must be properly determined.

For a drug displaying linear PK properties, the terminal phase seen in a graphical log-concentration versus time profile decreases in a straight line, independently of concentrations, and represents either the true elimination or the absorption of the drug (e.g., in the case of flip-flop pharmacokinetics). In noncompartmental analyses, $\mathrm{Kel}$ is estimated by linear regression from the slope of the terminal log-linear portion of the drug concentration versus time curve. The terminal elimination half-life is calculated from this constant as $\ln (2) /$ Kel. An improper

Corresponding Author: Murray P. Ducharme, PharmD, FCCP, FCP; Chief Science Officer, Cetero Research; 2000 Regency Parkway, Suite 255; Cary, NC, USA;

Email: murray.ducharme@cetero.com 
characterization of the PK parameters Kel and $\mathrm{T}^{1 / 2}$ will lead to a poor determination of the total exposure (i.e., AUCinf), an unacceptable characterization of the relative $\mathrm{BA}$ and therefore may lead to an incorrect BE conclusion. As such, certain criteria are useful in the determination of the Kel parameter. These include having at least three measurable concentration time-points in the terminal phase,[3] excluding the maximum observed concentration (Cmax) from the regression analysis and having a regression coefficient of at least 0.8. In addition to these common criteria, an additional important criterion that should be considered is the sampling scheme duration.

Simulations were thus undertaken to determine how the duration of a sampling scheme could affect the proper determination of the extent of exposure. The objectives of the work presented in this article are first to assess how the sampling duration affects the noncompartmental half-life or AUCinf determination and secondly to assess if these parameters should be excluded from the overall pivotal statistics based on the sampling duration to ensure adequate $\mathrm{BA}$ calculations and $\mathrm{BE}$ conclusions.

\section{METHODS - SIMULATIONS}

Concentration data for one thousand (1000) subjects receiving a single $100 \mathrm{mg}$ dose of a fictitious drug were simulated by Monte Carlo techniques in ADAPT 5®.[6] The model used to perform these simulations exhibited linear absorption (Ka) and elimination characteristics, included a central compartment $(\mathrm{Vc} / \mathrm{F})$, a peripheral compartment $(\mathrm{Vp} / \mathrm{F})$, a clearance from the central compartment $(\mathrm{CL} / \mathrm{F})$ and a distributional clearance $(\mathrm{CLd} / \mathrm{F})$. Concentration profiles were simulated assuming a normal distribution of the PK parameters. Figure 1 depicts the model used. The simulated concentration data were based on the following hypothetical PK population mean and variability values:

\begin{tabular}{ccc}
\hline Parameter & $\begin{array}{c}\text { Population } \\
\text { mean }\end{array}$ & $\begin{array}{c}\text { Inter-individual } \\
\text { CV\% }\end{array}$ \\
\hline $\mathrm{Ka}$ & 1 & 60 \\
$\mathrm{CL} / \mathrm{F}$ & 2.65 & 50 \\
$\mathrm{Vc} / \mathrm{F}$ & 8.5 & 30 \\
$\mathrm{CLd} / \mathrm{F}$ & 1.75 & 75 \\
$\mathrm{Vp} / \mathrm{F}$ & 16.7 & 75 \\
$\mathrm{~T} 1 / 2^{*}$ & \multicolumn{2}{c}{ 12 hours } \\
\hline
\end{tabular}

* $\mathrm{T}^{1} / 2$ was calculated based on the population parameters. Average $\mathrm{T}^{1} / 2$ presented.
The simulated concentration-time profiles included the following 21 sample time points: 0 (pre-dose), and 0.25, 0.5, 1, 1.5, 2, 3, 4, 5, 6, 8, 10, $12,14,16,18,21,24,30,36,48,60$ hours after dosing. Simulations were performed using a residual variability of $10 \%$. To determine the impact of a shorter sampling scheme, the concentration data at the end of the simulated profiles were progressively removed so that the duration of the entire sampling profile varied from 1 to 5 half-lives (i.e., 12 to 60 hours).

\section{NONCOMPARTMENTAL PK ANALYSIS}

The simulated subjects' data were then analyzed using a standard noncompartmental approach with $\mathrm{SAS} \otimes$ to obtain Kel, $\mathrm{T}^{1 / 2}$ and AUCinf. These noncompartmental PK parameters were recalculated for each different sampling scheme. Kel, $\mathrm{T}^{1} / 2$, and AUCinf were only set to missing if Kel was positive. Kel was calculated as the apparent first-order elimination rate constant calculated from a semi-log plot of the plasma concentration versus time curve. The parameter was calculated by linear least-squares regression analysis of the terminal log-linear phase.

\section{PRECISION AND BIAS}

True and known values for AUCinf and $\mathrm{T}^{1 / 2}$ for each subject were those obtained from the simulation in ADAPT 5® (i.e., ADAPT values were the true values). True AUCinf for each subject was calculated by dividing dose by $\mathrm{CL} / \mathrm{F}$ and true $\mathrm{T}^{1} 12$ was calculated from the parameters $\mathrm{CL} / \mathrm{F}$, CLd/F, Vc/F and Vp/F.[4] To determine how the duration of a sampling scheme affected the robustness of the calculated noncompartmental parameters, bias and precision as described by Sheiner[7] were evaluated for the noncompartmental parameters AUCinf and $T^{1 / 2}$. Precision of the noncompartmental AUCinf and T1/2 results with respect to the true results were assessed using the following formula: $100 *$ Absolute(noncompartment value - ADAPT value)/ADAPT value. Bias of the noncompartmental AUCinf and $\mathrm{T}^{1} / 2$ results with respect to the true results were evaluated using the following formula: $100 *$ (noncompartment value ADAPT value)/ADAPT value. Precision and bias judged acceptable were set a priori to $10 \%$ and $5 \%$, respectively. 


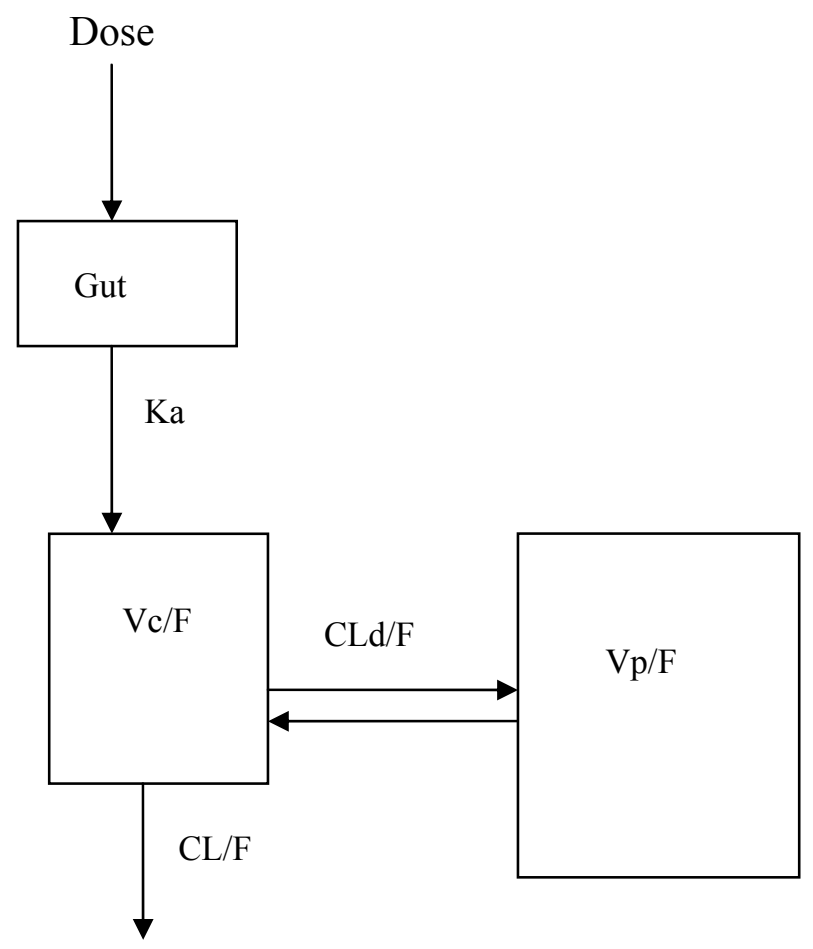

Figure 1. Model used to simulate concentrations

Simulated subjects with an absorption half-life that was longer than the terminal elimination halflife (i.e., flip-flop profiles) were excluded from the precision and bias calculation. This was done in order to avoid mixing different half-life types. The apparent terminal noncompartmental half-life would be a reflection of the absorption half-life and comparing this value to the known elimination halflife from the compartmental analysis would be wrong and would affect the precision and bias.

\section{BIOEQUIVALENCE STUDIES}

In addition to verifying the robustness (precision and bias) of the noncompartmental parameters, the impact of including or not data from subjects with unreliable noncompartmental AUCinf estimates on bioequivalence conclusions was evaluated using simulated studies. Using the same population PK parameters and variability parameters, thirty 2-way crossover studies each having 24 subjects were simulated. Ten studies per sampling scheme were derived based on sampling schemes that covered on average 2 half-lives (24 hours), 2.5 half-lives (30 hours) and 3 half-lives (36 hours). This resulted in some subjects in each study with potentially unreliable half-life estimates. Noncompartmental parameters were obtained in the same way as previously described and comparisons were made between the true values obtained from ADAPT $5 \AA$ and the noncompartmental estimates. Noncompartmental results were considered the test results while the ADAPT results were considered the reference results.

AUCinf were determined to be reliable or not using two different methods. The first method (Method 1) considered an AUCinf to be unreliable if it did not meet the acceptable a priori precision and bias of $10 \%$ and $5 \%$, respectively. The second method (Method 2) ignored the overall length of the sampling scheme as it considered only the duration of the sampling scheme over which Kel was determined. For this second method, an AUCinf was considered to be unreliable if the associated $\mathrm{T}^{1 / 2}$ was longer than the time span over which Kel was estimated as proposed by Purves. ${ }^{5}$

\section{ANALYSIS OF VARIANCE}

Analysis of variance (ANOVA) with Proc Mixed model in SAS ${ }^{\circledR}$ (Version 9.1.3) was performed to calculate the ratio and $90 \%$ confidence intervals 
(CI) on the ln-transformed AUCinf parameter (noncompartmental vs. true value). Three different ratios and $90 \%$ confidence intervals were calculated for each of the 30 simulated bioequivalence studies. Firstly, with all AUCinf included (i.e., without the removal of any unreliable AUCinf); secondly, with the removal of unreliable AUCinf as determined by Method 1 and thirdly, with the removal of unreliable AUCinf as determined by Method 2. The ANOVA model included sequence, formulation, and period as fixed effects and subject nested within sequence as a random effect. Sequence was tested using subject nested within sequence as the error term. These ANOVA were performed to determine the consequences on bioequivalence conclusions if unreliable results were included or not. Typically, bioequivalence is declared if the $90 \% \mathrm{CI}$ for parameters are within 80.00 to $125.00 \%$.
In these analyses, only the consequence on the AUCinf parameter is determined.

\section{RESULTS - PRECISION \& BIAS}

The precision and bias of the noncompartmental method to estimate the true PK parameters AUCinf and $\mathrm{T}^{1} 12$ are presented in Figure $2 \mathrm{~A}$ and B. Results are presented for sampling schemes covering 1 to 5 half-lives (i.e., 12 to 60 hours). As the sampling scheme duration was shortened from five half-lives to one half-life, the precision and bias in the noncompartmental PK parameters AUCinf and $\mathrm{T}^{1 / 2}$ deteriorated. The median precision and bias for AUCinf changed from $2.1 \%$ and $-0.1 \%$ to $14.0 \%$ and $-13.4 \%$. Similarly, the precision and bias for $\mathrm{T}^{1} 12$ deteriorated from $5.0 \%$ and $-1.4 \%$ to $65.5 \%$ and $-64.7 \%$, respectively.

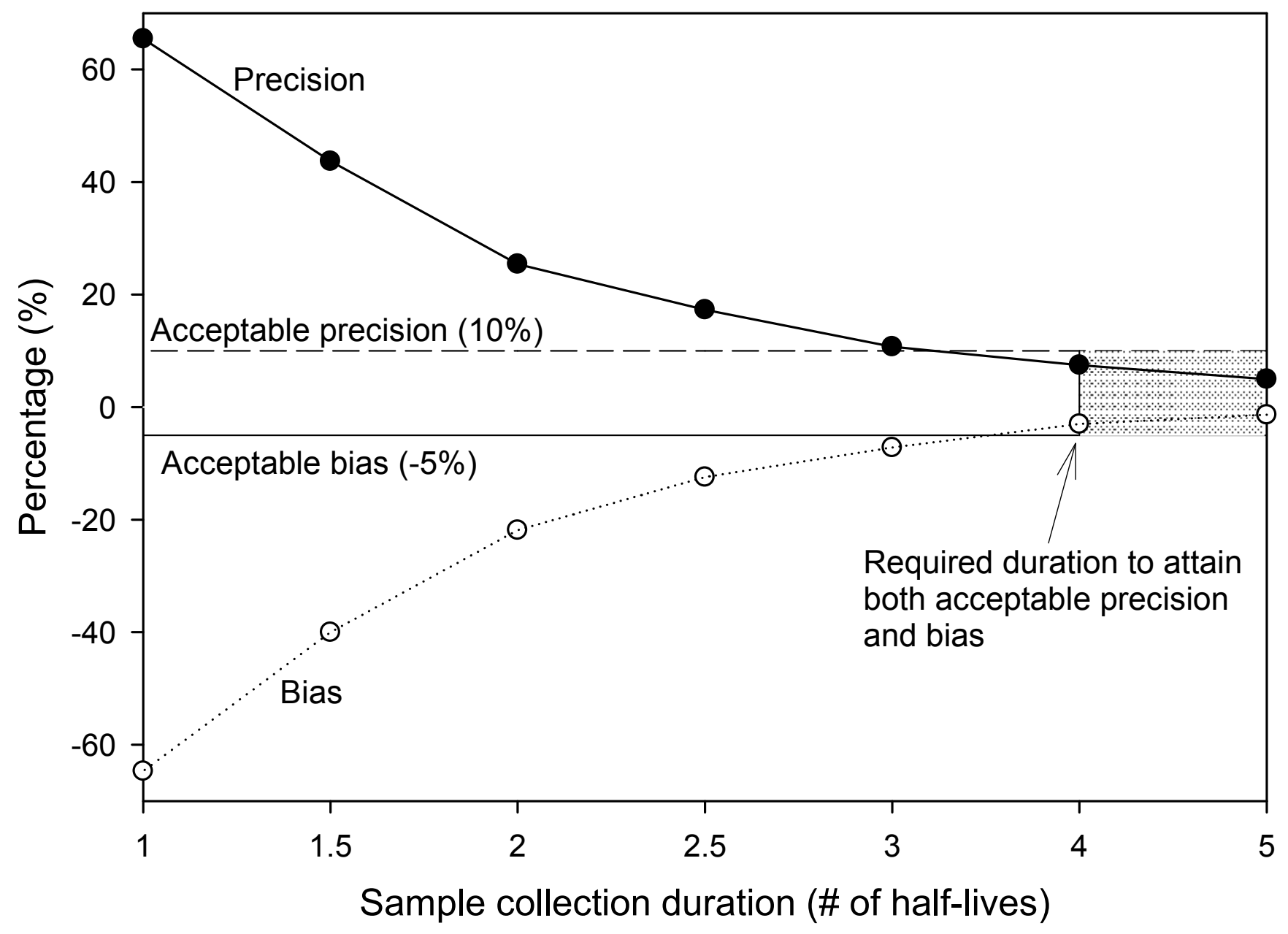

Figure 2A. Bias and precision of the half-life PK parameter 


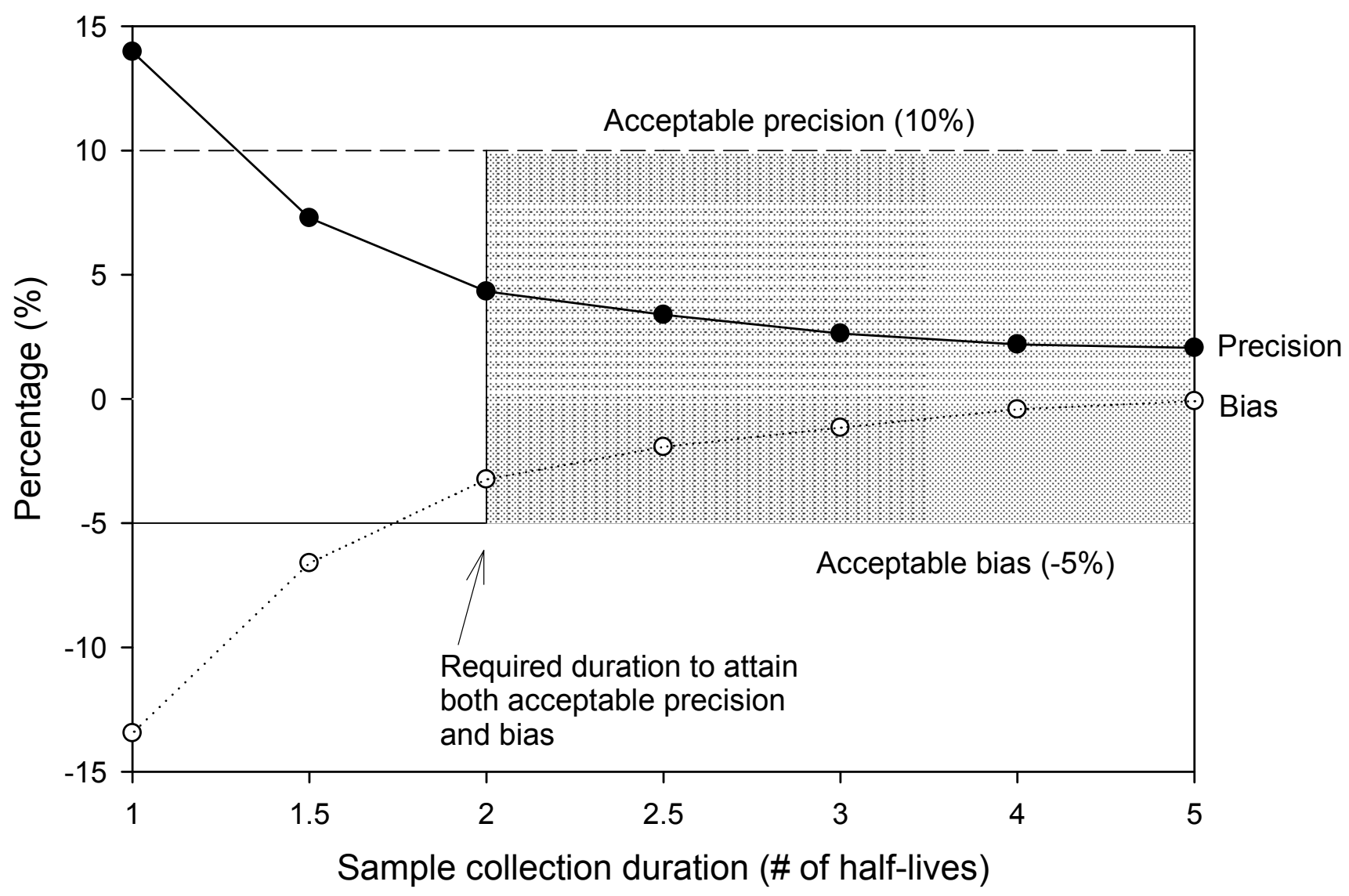

Figure 2B. Bias and precision of the AUCinf PK parameter

Results indicated that to have acceptable precision and bias in the noncompartmental AUCinf and $\mathrm{T}^{1 / 2}$ estimates, a sampling scheme that spanned over 2 and 4 half-lives was required, respectively. Sampling schemes covering less than 2 half-lives led to unreliable AUCinf estimates. In other words, AUCinf values were judged to be unreliable with Method 1 when they were calculated using half-life values observed to be longer than half of the overall sampling scheme.

Based on the bias results from all sampling schemes, the noncompartmental method estimated a shorter half-life than the true value (negative bias) and the shorter the sampling scheme, the shorter the noncompartmental half-life that was obtained.

\section{ANOVA - BIOEQUIVALENCE STUDIES}

The mean ratio comparing the noncompartmental AUCinf to the true AUCinf for the different sampling schemes are presented in Table 1 . Bioequivalence results from the 30 simulated studies are presented in Appendix 1. These results are presented with and without the unreliable AUCinf data based on the two already mentioned methods. Results indicated that if unreliable AUCinf were removed from within each BE study, the ratios were closer to $100 \%$ indicating that the noncompartmental estimates were closer to the true values. A longer sampling scheme reduced the number of unreliable AUCinf and decreased the magnitude of the improvement associated with removing these unreliable parameters. When 10 different BE studies were simulated per sampling scheme covering 2 (24 hours), 2.5 (30 hours) and 3 half-lives (36 hours), removing the unreliable AUCinf improved mean BE ratio by $6 \%$ (from $93.6 \%$ to $99.7 \%$ ), $4 \%$ (from $95.3 \%$ to $99.1 \%$ ) and $3 \%$ (from $96.9 \%$ to $99.6 \%$ ), respectively (Table 1 ). The AUCinf mean confidence interval ranges (Upper CI - Lower CI) for each sampling scheme including or not the unreliable values are depicted in Figure 3. On average, the $90 \%$ CI were narrower if the unreliable AUCinf were not included in the ANOVA. 
Table 1. Average BE ratio results from 10 studies ( $\mathrm{n}=24$ subjects in each study) per sampling scheme that covered on 2 half-lives ( 24 hours), 2.5 half-lives ( 30 hours) and 3 half-lives (36 hours) with and without unreliable AUCinf removed from analyses.

\begin{tabular}{cccc}
\hline Mean & $\begin{array}{c}\text { All AUCinf values } \\
\text { Ratio (\%) }\end{array}$ & $\begin{array}{c}\text { Without unreliable AUCinf } \\
\text { values (Method 1) Ratio (\%) }\end{array}$ & $\begin{array}{c}\text { Without unreliable AUCinf } \\
\text { values (Method 2) Ratio (\%) }\end{array}$ \\
\hline 24h sampling scheme & 93.55 & 99.72 & 99.13 \\
30h sampling scheme & 95.29 & 99.08 & 98.79 \\
36h sampling scheme & 96.90 & 99.57 & 99.27 \\
\hline
\end{tabular}

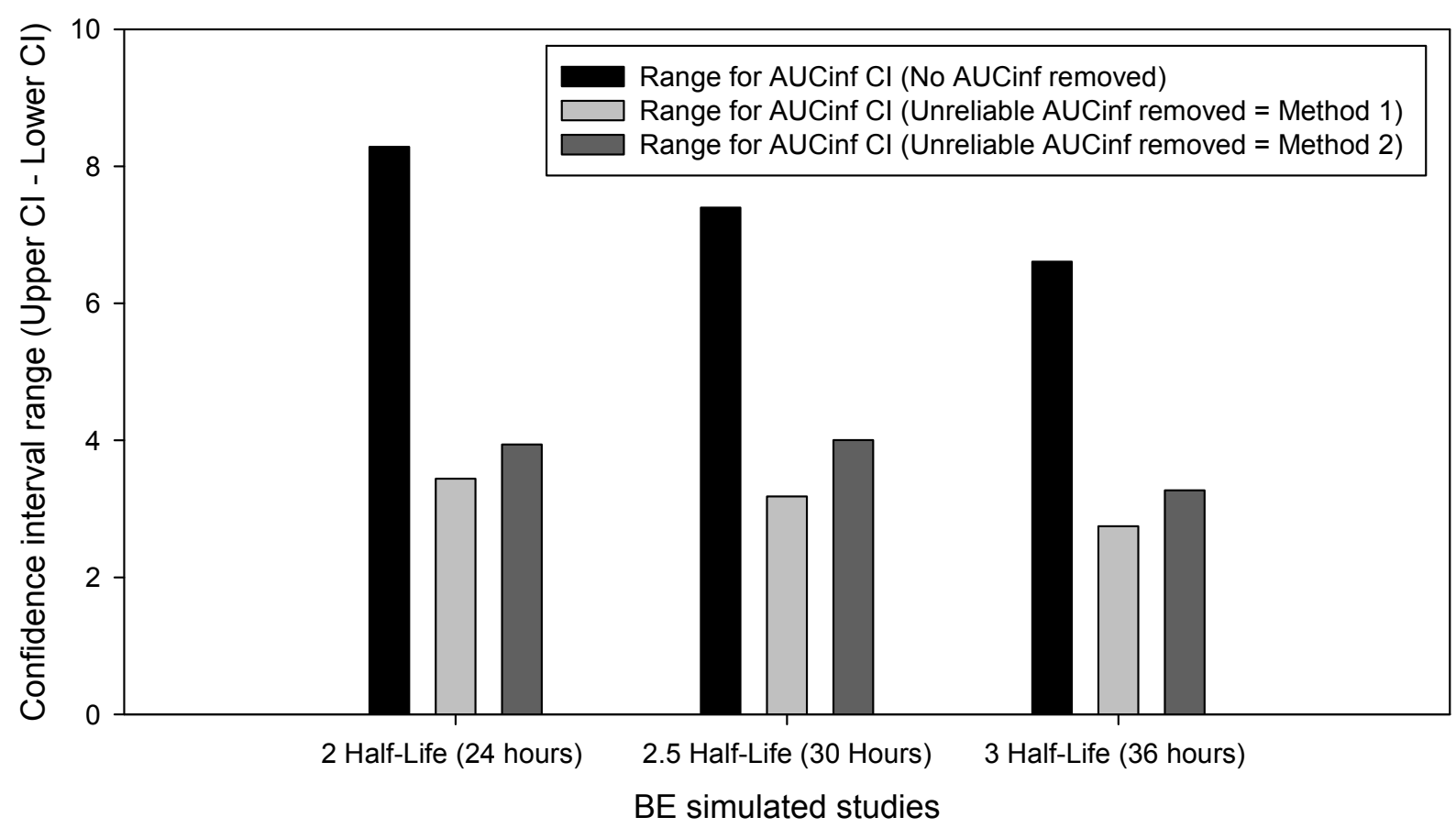

Note1: For Method 1, AUCinf based on half-lives greater than $50 \%$ of the sampling scheme duration was considered unreliable.

Note2: For Method 2, AUCinf based on half-lives greater than the sampling duration over which Kel was determined was considered unreliable

Figure 3. AUCinf confidence interval range for the different studies with and without unreliable AUCinf values

\section{DISCUSSION}

Pharmacokinetic parameters calculated using noncompartmental methods do not require much technical a priori expertise, and are considered to be the gold standard approach to be used for the vast majority of $\mathrm{BE}$ studies. It is generally appreciated though, that this method will only be reliable if the terminal half-life has been properly characterized. The absence of any formal guidance in this regard led us to perform simulations to determine the exact impact of the sampling scheme duration on the robustness of the half-life and AUCinf parameters. One thousand subjects were simulated with a sampling scheme covering 5 halflives and concentrations were removed at the end of the profile to obtain sampling schemes of shorter durations. This approach avoided having to simulate different subjects for each sampling scheme which would have added variability to the comparisons. By removing concentrations from the end of the profile, it was possible to directly compare the effect a shorter sampling scheme had on the precision and bias of the Kel and $\mathrm{T}^{1 / 2}$ 
determinations.

The noncompartmental method estimated a shorter half-life than the true value. The difference between the estimated and true half-life was more pronounced as the sampling scheme was shortened. This was expected, because with fewer samples collected during the elimination phase, the half-life determined from the noncompartmental method incorporated concentrations in the distribution phase instead of concentrations from only the elimination phase.

A shorter sampling scheme had a greater impact on the determination of the noncompartmental halflife than on the AUCinf parameter. This was also expected as AUCinf is dependent on both the halflife determination as well as AUC0-t. Therefore, any error on the half-life can only affect the extrapolated portion of AUCinf. With a sampling scheme spanning two half-lives, the extrapolated portion of AUCinf is only $25 \%$. Consequently, even a $20 \%$ bias of the half-life would only lead to a $5 \%$ error of the total AUCinf. The extrapolated portion is even smaller as the duration of the sampling scheme increases. This limits the impact a poor half-life determination could have on the AUCinf calculation.

For a pivotal PK study, a sampling scheme spanning greater than or equal to 4 half-lives was required to adequately characterize the half-life of a drug in order to maintain the bias and precision below $5 \%$ and $10 \%$, respectively. This is in line with the office of generic drugs of the US FDA requirements, but stricter than what is required in Canada or Europe (e.g., $80 \%$ of the AUCinf has to be observed, which is equivalent to a sampling duration spanning 2.3 half-lives). [8,9] Therefore, in drug development, one can have confidence in the half-life estimates of a drug if it was obtained from studies in which the sampling scheme duration spanned the equivalent of 4 half-lives or more.

In a bioequivalence setting, AUCinf precision and bias quickly deteriorated if the sampling scheme did not cover at least 2 half-lives, with bias being greater than $+/-5 \%$. When designing a $\mathrm{BE}$ study, the sample size of a study is often based on a predicted ratio that is $\pm 5 \%$. Therefore, a bias greater than $5 \%$ in the calculation of a PK parameter may add uncertainty and lead to a study that will be under-powered to prove BE. However, if subjects with AUCinf calculated using an unreliable half-life (eg, based on a value that is greater than half the sampling scheme or if the calculated $\mathrm{T}^{1 / 2}$ is less than the time span used in the calculation of Kel) were removed from the ANOVA results, ratios of AUCinf improved and were closer to the true ratio of $100 \%$. In addition, the average confidence intervals for AUCinf tightened if the unreliable subject's data were removed from the ANOVA. Improvement in the ratios and confidence intervals for AUCinf was noted even with the loss of degrees of freedom due to the removal of data from subjects with AUCinf considered unreliable. Therefore, the loss of degrees of freedom was more than offset by the removal of variability caused by keeping these unreliable values.

Our results are not meant to salvage poorly designed bioequivalence studies by stating that sampling schemes could span 2 half-lives and that this is enough for acceptable study conclusions. However, all studies are based on expected mean values and a certain amount of inter-subject and intra-subject variability. Occasionally, results will be unexpected or an individual subject's results will fall outside the anticipated limit of variability. Our results suggest that the additional suggested criterion should be set a priori even if the mean results (e.g., AUCt/AUCinf ratio) are within accepted criteria set by the regulatory agencies. This will provide confidence intervals that are closer to the truth.

Increased bias in the AUCinf PK parameter with decreasing time span for Kel determination was previously noted by Purves. [5] This author reported that the variance of the extrapolation in the AUC estimates rapidly increased if the time span for Kel determination was less than the half-life and suggested that the Kel determination should be obtained from a regression that spanned at least two half-lives. Other authors also determined the impact of large bias in the extrapolation portion of the AUCinf or area under the moment curve (AUMC) parameters.[10,11] However, their work focused on the impact on the mean residence time (MRT). The analyses presented in this paper aimed to determine the duration of a sampling scheme that was required to obtain robust half-life and AUCinf results. In addition, this work demonstrated the negative consequences of maintaining unreliable AUCinf on $\mathrm{BE}$ conclusions.

As previously mentioned, many scientists in industry and academia use different criteria to avoid 
having an improper characterization of the PK parameters Kel, $\mathrm{T}^{1} / 2$ and AUCinf. These typically include determining Kel with at least three terminal time points without including the Cmax, and having a regression coefficient $\left(\mathrm{R}^{2}\right)$ of at least 0.8 . To precisely determine the impact of the sampling scheme duration on the PK parameters Kel, $\mathrm{T}^{1 / 2}$ and AUCinf, these criteria were not used. However, it is possible that by adding these criteria, the influence of the sampling scheme duration may be further reduced. Therefore, the noncompartmental parameters were recalculated with these criteria added to determine if the criterion of the sampling scheme had the same impact on the conclusions. Although precision and bias were slightly improved when these criteria were used in addition to sampling scheme duration, conclusions remained identical.

Two methods were used to identify the unreliable AUCinf values and verify the impact of removing them on the $90 \%$ confidence intervals of bioequivalence studies. Based on the BE results, both methods were comparable and helped to obtain results that were closer to the truth and reduce the overall uncertainty. A difference that exists between the two methods is that the criterion of half the sampling scheme is identical for all subjects within a study making it easier to use as a criterion, while the time span of the Kel is different for every subject.

\section{CONCLUSIONS}

The results of this study suggest that the sampling duration of any pivotal PK study be at least 4 times the average measured half life in order to have confidence in the reported half-life values and thereby in the extent of exposure parameter AUCinf. The analyses also suggest that individual subject's PK parameters AUCinf, kel and $\mathrm{T}^{1 / 2}$ should be removed from the pivotal statistical analysis (i.e. such as in a BE study) when their associated calculated half-life is longer than half of the total sampling interval, as this will reduce the overall uncertainty and provide results closer to the true values.

\section{REFERENCES}

1. Bioavailability and bioequivalence requirements. Code of Federal Regulations Title 21, Volume 5, Pt. 320.1. Revised April 1, 2009. Access May 6, 2010.

2. Niazi, S.K. Handbook of bioequivalence testing. Informa Healthcare, New York, NY, 2007.

3. Guidance for Industry. Bioavailability and bioequivalence studies for orally administered drug products - general considerations. U.S. Department of Health and Human Services Food and Drug Administration Center for Drug Evaluation and Research (CDER). March 2003.

4. Gibaldi $\mathrm{M}$ and Perrier D. Pharmacokinetics. $2^{\text {nd }}$ ed (revised and expanded). Informa Healthcare, New York, NY, 2007.

5. Purves RD. Bias and variance of extrapolated tails for area-under-the-curve (AUC) and area-underthe-moment-curve (AUMC). J Pharmacokinet Biopharm. 1992 Oct;20(5):501-10.

6. D'Argenio, D.Z., A. Schumitzky and X. Wang. ADAPT 5 User's Guide: Pharmacokinetic/Pharmacodynamic Systems Analysis Software. Biomedical Simulations Resource, Los Angeles, 2009.

7. Sheiner LB, Beal SL. Some suggestions for measuring predictive performance. J Pharmacokinet Biopharm. 1981 Aug;9(4):503-12.

8. Guidance for Industry. Conduct and analysis of bioavailability and bioequivalence studies - Part A: Oral dosage formulations used for systemic effects. Canada Health Products and Food Branch Guidance Document. 1992.

9. Draft guideline on the investigation of bioequivalence. European Medicines Agency; Committee for Medicinal Products for Human use (CHMP). London, 24 July 2008.

10. Riegelman S, Collier P. The application of statistical moment theory to the evaluation of in vivo dissolution time and absorption time. $\mathrm{J}$ Pharmacokinet Biopharm. 1980 Oct;8(5):509-34.

11. Purves RD. Numerical estimation of the noncompartmental pharmacokinetic parameters variance and coefficient of variation of residence times. J Pharm Sci. 1994 Feb;83(2):202-5. 
APPENDIX 1. BE results from 30 studies ( $n=24$ subjects in each study) with and without unreliable AUCinf removed from analyses

\section{4h sampling scheme}

\begin{tabular}{|c|c|c|c|c|c|c|c|c|c|}
\hline \multirow[b]{2}{*}{ Study } & \multicolumn{3}{|c|}{ All AUCinf values } & \multicolumn{3}{|c|}{ Without unreliable AUCinf values (Method 1) } & \multicolumn{3}{|c|}{ Without unreliable AUCinf values (Method 2) } \\
\hline & Ratio (\%) & Lower CI (\%) & Upper CI (\%) & Ratio (\%) & Lower CI (\%) & Upper CI(\%) & Ratio (\%) & Lower CI (\%) & Upper CI(\%) \\
\hline 1 & 92.87 & 87.49 & 98.57 & 100.50 & 98.12 & 102.93 & 98.86 & 96.60 & 101.18 \\
\hline 2 & 95.91 & 92.84 & 99.08 & 99.84 & 97.51 & 102.22 & 99.05 & 96.88 & 101.28 \\
\hline 3 & 93.51 & 90.19 & 96.95 & 99.50 & 98.55 & 100.46 & 99.18 & 97.77 & 100.61 \\
\hline 4 & 95.80 & 93.40 & 98.27 & 101.76 & 98.95 & 104.65 & 100.48 & 98.12 & 102.89 \\
\hline 5 & 88.67 & 84.20 & 93.38 & 99.45 & 97.93 & 101.00 & 99.41 & 97.21 & 101.66 \\
\hline 6 & 96.91 & 93.25 & 100.71 & 99.41 & 97.89 & 100.96 & 98.54 & 96.46 & 100.67 \\
\hline 7 & 92.58 & 90.10 & 95.13 & 97.47 & 96.40 & 98.55 & 97.39 & 95.23 & 99.60 \\
\hline 8 & 96.89 & 93.21 & 100.71 & 100.24 & 98.73 & 101.77 & 99.86 & 98.69 & 101.04 \\
\hline 9 & 88.28 & 80.07 & 97.33 & 99.00 & 97.04 & 100.99 & 99.45 & 97.78 & 101.14 \\
\hline 10 & 94.09 & 90.43 & 97.90 & 100.05 & 99.05 & 101.05 & 99.09 & 97.07 & 101.15 \\
\hline Mean & 93.55 & 89.52 & 97.80 & 99.72 & 98.02 & 101.46 & 99.13 & 97.18 & 101.12 \\
\hline
\end{tabular}

\section{0h sampling scheme}

\begin{tabular}{|c|c|c|c|c|c|c|c|c|c|}
\hline \multirow[b]{2}{*}{ Study } & \multicolumn{3}{|c|}{ All AUCinf values } & \multicolumn{3}{|c|}{ Without unreliable AUCinf values (Method 1) } & \multicolumn{3}{|c|}{ Without unreliable AUCinf values (Method 2) } \\
\hline & Ratio (\%) & Lower CI (\%) & Upper CI (\%) & Ratio (\%) & Lower CI (\%) & Upper CI(\%) & Ratio (\%) & Lower CI (\%) & Upper CI(\%) \\
\hline 11 & 96.06 & 91.39 & 100.98 & 99.56 & 97.65 & 101.51 & 99.27 & 96.62 & 102.00 \\
\hline 12 & 95.91 & 92.84 & 99.08 & 98.98 & 96.98 & 101.03 & 97.93 & 95.42 & 100.51 \\
\hline 13 & 93.59 & 88.60 & 98.86 & 99.20 & 98.03 & 100.38 & 99.20 & 98.03 & 100.38 \\
\hline 14 & 98.84 & 97.23 & 100.47 & 99.97 & 98.59 & 101.37 & 99.00 & 97.39 & 100.63 \\
\hline 15 & 93.29 & 89.43 & 97.32 & 98.73 & 96.62 & 100.89 & 99.69 & 96.16 & 101.28 \\
\hline 16 & 95.85 & 92.36 & 99.48 & 99.03 & 97.44 & 100.64 & 99.02 & 97.69 & 100.38 \\
\hline 17 & 95.07 & 92.92 & 97.28 & 97.38 & 96.28 & 98.49 & 96.67 & 95.21 & 98.15 \\
\hline 18 & 97.55 & 94.78 & 100.39 & 99.07 & 97.33 & 100.84 & 97.93 & 94.71 & 101.26 \\
\hline 19 & 91.78 & 85.93 & 98.03 & 99.05 & 97.23 & 100.90 & 99.16 & 96.88 & 101.48 \\
\hline 20 & 94.94 & 91.24 & 98.80 & 99.86 & 98.90 & 100.84 & 100.00 & 98.98 & 101.04 \\
\hline Mean & 95.29 & 91.67 & 99.07 & 99.08 & 97.51 & 100.69 & 98.79 & 96.71 & 100.71 \\
\hline
\end{tabular}




\section{6h sampling scheme}

All AUCinf values $\quad$ Without unreliable AUCinf values (Method 1) Without unreliable AUCinf values (Method 2)

\begin{tabular}{|c|c|c|c|c|c|c|c|c|c|}
\hline \multirow[b]{2}{*}{ Study } & \multirow[b]{2}{*}{ Ratio (\%) } & & & \\
\hline & & Lower CI (\%) & Upper CI (\%) & Ratio (\%) & Lower CI (\%) & Upper CI(\%) & Ratio (\%) & Lower CI (\%) & Upper CI(\%) \\
\hline 21 & 96.10 & 91.94 & 100.44 & 99.26 & 97.51 & 101.04 & 99.45 & 97.65 & 101.29 \\
\hline 22 & 95.91 & 92.84 & 99.08 & 99.30 & 97.33 & 101.30 & 99.58 & 97.56 & 101.65 \\
\hline 23 & 95.39 & 91.21 & 99.77 & 100.33 & 99.35 & 101.32 & 100.00 & 99.01 & 101.01 \\
\hline 24 & 99.42 & 97.39 & 101.50 & 100.37 & 99.29 & 101.45 & 99.90 & 98.49 & 101.33 \\
\hline 25 & 96.71 & 92.71 & 100.88 & 99.37 & 97.50 & 101.28 & 99.73 & 97.90 & 101.60 \\
\hline 26 & 98.01 & 94.50 & 101.65 & 99.73 & 98.68 & 100.79 & 100.04 & 98.95 & 101.16 \\
\hline 27 & 97.40 & 96.17 & 98.66 & 98.31 & 97.14 & 99.50 & 97.83 & 96.85 & 98.81 \\
\hline 28 & 97.84 & 95.79 & 9.92 & 100.13 & 98.80 & 101.49 & 98.44 & 96.03 & 100.91 \\
\hline 29 & 94.42 & 89.73 & 99.35 & 99.04 & 97.55 & 100.55 & 98.12 & 95.57 & 100.74 \\
\hline 30 & 97.82 & 94.43 & 101.54 & 99.83 & 98.88 & 100.78 & 99.64 & 98.53 & 100.76 \\
\hline Mean & 96.90 & 93.67 & 91.28 & 99.57 & 98.20 & 100.95 & 99.27 & 97.65 & 100.93 \\
\hline
\end{tabular}

